\title{
Channel Modelling for Multiprobe Over-the-Air MIMO Testing
}

\author{
Pekka Kyösti, Tommi Jämsä, and Jukka-Pekka Nuutinen \\ Elektrobit Corporation, Wireless Business Segment, Tutkijantie 8, 90590 Oulu, Finland \\ Correspondence should be addressed to Pekka Kyösti, pekka.kyosti@elektrobit.com
}

Received 28 December 2011; Accepted 20 March 2012

Academic Editor: Moray Rumney

Copyright ( 2012 Pekka Kyösti et al. This is an open access article distributed under the Creative Commons Attribution License, which permits unrestricted use, distribution, and reproduction in any medium, provided the original work is properly cited.

\begin{abstract}
This paper discusses over-the-air (OTA) test setup for multiple-input-multiple-output (MIMO) capable terminals with emphasis on channel modelling. The setup is composed of a fading emulator, an anechoic chamber, and multiple probes. Creation of a propagation environment inside an anechoic chamber requires unconventional radio channel modelling, namely, a specific mapping of the original models onto the probe antennas. We introduce two novel methods to generate fading emulator channel coefficients; the prefaded signals synthesis and the plane wave synthesis. To verify both methods we present a set of simulation results. We also show that the geometric description is a prerequisite for the original channel model.
\end{abstract}

\section{Introduction}

To meet the increased consumer demand on high data rate applications_-such as music and video downloading, web browsing, and multimedia sharing-multiantenna technology will be widely utilized in mobile terminals near future. New standards such as the 3rd Generation Partnership Project (3 GPP) High Speed Downlink Packet Access (HSDPA), Long Term Evolution (LTE), LTE-Advanced, and IEEE $802.16 \mathrm{~m}$ specify multiple antenna technology also at mobile terminal, and IEEE $802.11 \mathrm{n}$ and 802.11 ac specify multiple antennas in Wireless Local Area Network (WLAN) devices. Multiple-input-multiple-Output (MIMO) technology in wide sense covers any multi antenna technique, such as spatial multiplexing, beam forming, and spatial diversity. MIMO offers significant increases in data throughput, quality of service (QoS), and cell coverage without additional bandwidth or transmit power. Communication performance is improved by exploiting the characteristics of the propagation channel in which the device is operating.

In MIMO systems, spatial correlation plays a key role. It depends on both antenna and propagation characteristics. Neither can determine the correlation alone. Therefore, it is necessary to include both antenna and propagation effects at the same time when testing multiantenna terminals. MIMO over-the-air (OTA) testing provides solution for that demand. Due to the complexity of multiple antenna setups, flexible, fast, and accurate testing solution is needed to speed up the development process and to ensure the real performance of the terminal. Foegelle [1] identified the need for a new approach for OTA testing of multiantenna terminals to complement the existing OTA test methodologies for single antenna terminals.

Recently, several different MIMO OTA test methodologies have been proposed in research and standardization forums. European Cooperation in Science and Technology (COST) action COST2100 [2] has been finished and the final report will be published soon [3]. Follower of COST2100, new COST IC1004 action continues the work, and the final results are expected in year 2015 [4]. 3GPP MIMO OTA Study Item is almost finished, and the work will potentially continue in a Work Item [5]. CTIA [6] discusses MIMO OTA as well. In all of these groups, three fundamentally different categories of methodologies have been proposed. The methodologies in the first category are based on anechoic chamber and a number of probe antennas, in which the signals are controlled by for example a fading emulator. The second category provides angular dispersion of multipath signals via a reverberation chamber. The power angular spectrum is 3D uniform. By using an external fading emulator, longer delay spread can be achieved. Third, multistage methodology is based on a number of measurement steps to evaluate the OTA performance. In the first stage the complex antenna pattern is measured by a 
traditional anechoic chamber-based antenna measurement system, single-input-single-output (SISO) OTA. The second stage combines the antenna pattern information and the channel model to calculate the MIMO correlation matrices and antenna power imbalances, which are implemented in a channel emulator to make a conductive measurement or theoretical capacity calculation. The two first MIMO OTA methodologies provide a possibility to measure the true mobile terminal performance without using artificial cabling in the test setup. On the contrary, conductive test requires an RF cable connection, which affect the terminal RF and antenna performance.

MIMO OTA testing based on an anechoic chamber and a fading emulator (the first methodology) enables true evaluation of the end-user experience of the final product against realistic radio channel conditions. It also makes it possible to change the channel model (scenario) flexibly via software, for example signal angle-of-arrival (AoA) and angle spread are controllable. All critical parts of the mobile terminal design (antennas, RF front end, baseband processing) are tested at once. In traditional conductive testing, as in LTE conformance tests, only certain baseband processing is tested with predefined correlation characteristics, which omits the antenna effect in testing.

Anechoic chamber-based MIMO OTA enables to test off-the-shelf products (i.e., end products) in equivalent radio propagation conditions to provide unquestionable comparisons between the devices under test. In practice, the performance differences between "golden samples" and mass products may be large, thus there is a need for end device test system that can be used for mass production device testing without any cable connections.

This paper describes the anechoic chamber and fading emulator-based MIMO OTA test methodology. In Section 2 the overall system is described. Section 3 discusses and proposes channel models for MIMO OTA. Section 4 describes how the desired channel model is mapped onto the limited number of probe antennas. Two novel methods, namely, prefaded signals synthesis and plane wave synthesis, are explained. Section 5 shows simulation results, and Section 6 concludes this paper.

\section{Anechoic Chamber and Fading Emulator-Based System}

The MIMO OTA test setup, originally described in [7], is based on a fading emulator, an anechoic chamber and a number of OTA antennas. The purpose of the setup is to reproduce time variant electromagnetic field around the device under test (DUT) imitating the target MIMO radio channel model, as accurately as possible. The idea is to apply widely approved channel models like TGn model [8], SCM(E) [9], WINNER [10], or IMT-Advanced [11] on a radiated testing of DUT performance.

The components of OTA performance test setup are illustrated in Figure 1. DUT is in the centre of the anechoic chamber, in an area called test zone or test area, and transmitting antennas are arranged, for example, uniformly



FIGURE 1: Fading emulator and anechoic chamber based MIMO OTA test set-up.

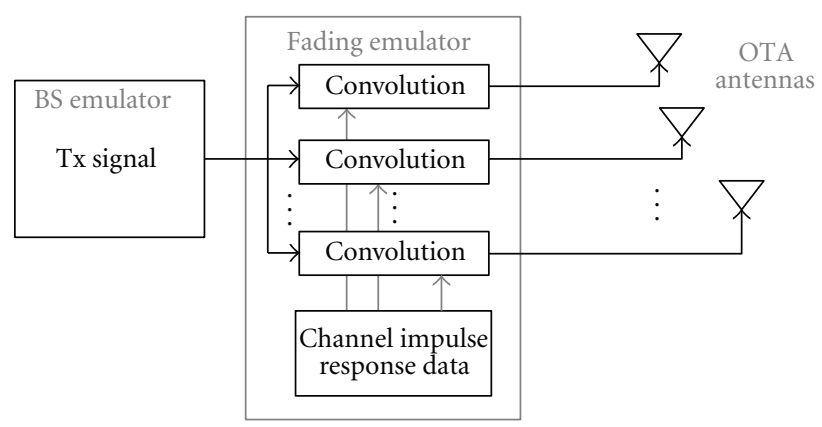

Figure 2: Convolution of Tx signal and radio channel impulse response data in the fading emulator.

around the DUT in two or three dimensions. Each of the transmitting antennas is connected to an output port of the fading emulator. Typically a power amplifier, between a fading emulator output and an OTA probe, is required to compensate path loss between the OTA probe and DUT. The communication tester (BS emulator) creates the test signal, which is fed to the multichannel fading emulator. The emulator creates the multipath environment including path delays, Doppler spread and fast fading. A fading emulator performs convolution of $\mathrm{Tx}$ signals with channel model impulse responses as described in Figure 2. The channel models containing directions of departures and arrivals are mapped to emulator so that model allocation corresponds to physical antenna installation in the chamber. Phantom heads, hands, and so forth can be easily added to the measurement. DUT is assumed to be in the far field region of the OTA antenna radiation.

In the actual MIMO OTA test of a multiantenna terminal an appropriate performance metric, for example throughput, is collected as a figure of merit. DUT may be rotated around one, two, or three rotation axis and the final performance may be the average performance over different DUT orientations. The performance averaged over a number of DUT orientations is effectively different to, for example, the performance in $3 \mathrm{D}$ isotropic scattering environment of a reverberation chamber. From mathematics it is well known that in general case an average of function values is different to a function value of averaged function argument. 


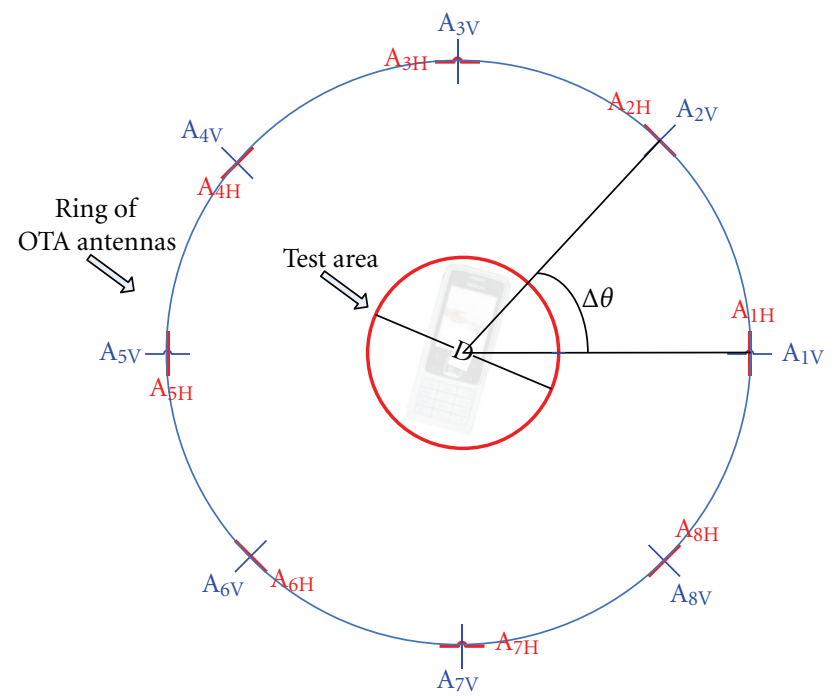

Figure 3: Ring of OTA antennas and the test zone.

Typically both downlink and uplink transmissions are required in the measurement. An uplink communication antenna is located inside the anechoic chamber so that it does not cause interference problems to downlink. A proper uplink antenna would be a circularly polarized antenna with high directivity. The antenna is connected to a base station (BS) emulator with a coaxial cable. In the simplest case no fading or multipath effects are emulated in the uplink. In time division duplex (TDD) systems the uplink radio channel should be reciprocal to downlink, but TDD case is not discussed in this paper. A system diagram for TDD MIMO OTA test setup is presented in [12].

OTA antennas must be dual-polarized if also polarization dimension of the radio channel is considered. Both elements of a single OTA antenna, radiating orthogonally polarized signals, have to be connected to different fading emulator outputs. This will guarantee independent fading on different polarizations as specified, for example in SCM and WINNER channel models $[10,13]$. An example layout of eight dualpolarized OTA antennas is illustrated in Figure 3 with uniformly spaced probes on a circle, resulting to angular spacing $\Delta \theta=45^{\circ}$.

The example layout in Figure 3 is of 2D setup. Most of the standardized radio channel models are two dimensional (2D) in the sense that they use only geometrical $x y$-coordinates (azimuth plane) [14]. Elevation dimension has been left out because the power angular spectrum is typically confined close to the horizon. The MIMO OTA setup can be extended to $3 \mathrm{D}$ by installing antennas on, for example, cylindrical or spherical formation. A 3D addendum for IMT-advanced channel models is given in [14].

The number of required OTA antennas has been discussed in $[15,16]$ and in various COST 2100 contributions. The final formula is still missing, but the number depends on the DUT size, the centre frequency, the channel model, the
OTA antenna locations, and the acceptable error level. The following rule of thumb is given in [15]:

$$
K=2\left\lceil\frac{\pi D}{\lambda}\right\rceil+1,
$$

where $K$ is a number of OTA antennas, [ $[$ denotes round up operation, $D$ is diameter of the test area as in the Figure 3, and $\lambda$ is the wavelength.

In a practical setup there may be unintentional reflections of transmitted downlink signals from other OTA antennas. Preliminary measurements of [17] indicate that the reflections are not a serious problem for the field synthesis. The average scattering level was $35 \mathrm{~dB}$ below the line-of-sight path power with a directive Vivaldi type of OTA antenna design. In the measurement the observed frequency range was $1.35-7.25 \mathrm{GHz}$ and the distance from OTA antenna to the centre of the test area was at minimum 1 metre.

\section{Geometric Channel Models}

There exists a high variety of different kinds of MIMO radio channel models. An overview of models is given in [18]. MIMO radio channel models typically have to cover all the four dimensions of the radio channel, namely, time, frequency, space, and polarization. Thus the models are time variant, wideband, double directional, and polarimetric. The two most popular classes of MIMO channel models are socalled geometry-based models and correlation matrix based models. Models for example in $[10,11,13]$ belong to the geometric family and in [8] to the family of correlation matrix based models. In many cases also the correlation matrix based models have geometric description as an initial starting point. This is the case also in TGn model.

Geometry based modelling enables separation of propagation and antennas. Antenna geometries and field patterns can be defined independent of propagation parameters. Channel realisations are generated with the geometrical principle by summing contributions of rays (plane waves) with specific small scale parameters like for example delay, power, angle of arrival (AoA), and angle of departure (AoD). Superposition results to correlation between antenna elements and temporal fading with geometry-dependent Doppler spectrum [10].

A widely approved concept in spatial channel modelling is the concept of "cluster." A number of rays constitute a cluster. In a common terminology the cluster is understood as a propagation path diffused in space, either or both in delay and angle domains. Typically a cluster is composed of a fixed number of rays (sub paths) and has a specific shaped power angular spectrum, for example, Laplacian function, defined by nominal AoA/AoD and angular spread of arrival and departure (ASA/ASD). A physical propagation mechanism creating clusters is reflection, scattering, or diffraction on a limited sized object in the physical environment, for example on a corner of a building. A cluster can be composed of, for example, a high number of scattering points on surface of a building. Each scatterer has characteristics like AoA, AoD, and complex attenuation coefficients. A single 
ray of geometric model represents scattering (or reflection or diffraction) on a single scattering point.

A geometry-based channel model can be composed from the following propagation parameters: cluster powers, delays, nominal arrival and departure angles, and angle spreads of clusters on both arrival and departure ends, and cluster cross-polarization power ratios (XPRs). In addition, information of the receiver and transmitter antenna arrays including both array geometry and antenna field patterns is required. (Note that Rx antennas are not specified in MIMO OTA.) Also either the terminal velocity vector or the cluster Doppler frequency components have to be defined.

In the following we define a system model for horizontal plane (2D) MIMO radio channel based on [10, 13]. Transfer matrix of the MIMO channel is

$$
\mathbf{H}(t, \tau)=\sum_{l=1}^{L} \mathbf{H}_{l}(t, \tau) .
$$

It is composed of antenna array response matrices $\mathbf{F}_{t x}(\mathrm{Tx})$, $\mathbf{F}_{r x}(\mathrm{Rx})$, and impulse response matrices $\mathbf{h}_{l}$ for rays $l$ as follows:

$$
\mathbf{H}_{l}(t, \tau)=\iint \mathbf{F}_{r x}^{T}(\varphi) \mathbf{h}_{l}(t, \tau, \phi, \varphi) \mathbf{F}_{t x}(\phi) d \varphi d \phi
$$

The channel impulse response of the $l$ th ray is a $2 \times 2$ polarimetric matrix

$$
\begin{aligned}
\mathbf{h}_{l}(t, \tau, \phi, \varphi) \\
\quad=\left[\begin{array}{ll}
\alpha_{l}^{V V}(t) & \alpha_{l}^{V H}(t) \\
\alpha_{l}^{H V}(t) & \alpha_{l}^{H H}(t)
\end{array}\right] \delta\left(\tau-\tau_{l}\right) \delta\left(\phi-\phi_{l}\right) \delta\left(\varphi-\varphi_{l}\right) .
\end{aligned}
$$

Now we introduce the clustered structure of a propagation channel and replace ray index $l$ with cluster index $n$ and cluster sub path index $m$. The channel coefficient for a channel from Tx antenna element $s$ to Rx antenna element $u$ for the cluster $n$ can be modelled as

$$
\begin{aligned}
& H_{u, s, n}(t, \tau) \\
& =\sum_{m=1}^{M}\left[\begin{array}{l}
F_{r x, u, V}\left(\varphi_{n, m}\right) \\
F_{r x, u, H}\left(\varphi_{n, m}\right)
\end{array}\right]^{T} \mathbf{A}\left[\begin{array}{l}
F_{t x, s, V}\left(\phi_{n, m}\right) \\
F_{t x, s, H}\left(\phi_{n, m}\right)
\end{array}\right] \\
& \cdot \exp \left(j 2 \pi v_{n, m} t\right) \delta\left(\tau-\tau_{n}\right),
\end{aligned}
$$

where $v_{n, m}$ is the Doppler shift of sub path $n, m$ and $2 \times 2$ polarization matrix of scattering coefficients is

$$
\mathbf{A}=\left[\begin{array}{ll}
\alpha_{n, m}^{V V} & \alpha_{n, m}^{V H} \\
\alpha_{n, m}^{H V} & \alpha_{n, m}^{H H}
\end{array}\right] .
$$

For example, the coefficient $\alpha_{n, m}^{H V}$ contains phase rotation and attenuation for vertically polarized incident wave and horizontally polarized scattered wave of ray $m$ of cluster $n$. Coefficients $\alpha$ are modelled as time invariant in cluster based geometric models. Phases are typically random and amplitudes are determined by the XPR. Random phases result always to elliptical polarization. For a line-of-sight path with linear polarization the off-diagonal elements of $\mathbf{A}$ are zeros and diagonal elements have equal phase.

The presented $2 \mathrm{D}$ model can be extended to $3 \mathrm{D}$, by interpreting angles $\varphi$ and $\phi$ as composed of azimuth and elevation components like, for example, $\varphi=\left(\varphi_{a z}, \varphi_{e l}\right)$. Elevation angular parameters to extend the model are; elevation angle of arrival (EoA) and departure (EoD), and elevation angle spread of arrival (ESA) and departure (ESD).

Cross-polarization power ratio (XPR) has different definitions. Here we follow and clarify the definition of [10], where XPR is a pure propagation parameter. XPR for vertically polarized $\mathrm{Tx}$ signals is

$$
\mathrm{XPR}_{V}=\frac{S^{V V}}{S^{H V}}=\frac{\left|\alpha^{V V}\right|^{2}}{\left|\alpha^{H V}\right|^{2}},
$$

respectively, XPR for horizontally polarized Tx signals is

$$
\mathrm{XPR}_{H}=\frac{S^{H H}}{S^{V H}}=\frac{\left|\alpha^{H H}\right|^{2}}{\left|\alpha^{V H}\right|^{2}},
$$

where

(a) $S^{V V}$ is the coefficient for scattered power on $V$ polarization and incident power on $V$-polarization of an interacting object (cluster),

(b) $S^{V H}$ is the coefficient for scattered power on $V$ polarization and incident power on $H$-polarization of an interacting object (cluster),

(c) $S^{H V}$ is the coefficient for scattered power on $H$ polarization and incident power on $V$-polarization of an interacting object (cluster),

(d) $S^{H H}$ is the coefficient for scattered power on $H$ polarization and incident power on $H$-polarization of an interacting object (cluster).

Even though $\mathrm{XPR}_{V}$ and $\mathrm{XPR}_{H}$ are defined separately, it is often assumed that $\mathrm{XPR}_{V}=\mathrm{XPR}_{H}$. This is the assumption, for example, in $[10,11]$.

3.1. Channel Model Prerequisites for MIMO OTA. Geometric description of the propagation and the separation of antennas and propagation are essential requirements for the basic radio channel model to be reconstructed to a MIMO OTA test setup. Pure correlation matrix models are not realizable with a MIMO OTA setup, because they don not fulfil these prerequisites. At least the DUT end of the channel model has to be specified by angular propagation parameters. If some antenna characteristics are embedded to the channel model, which is the case in pure correlation matrix-based model, the model itself assumes some DUT antennas. Thus it is not feasible to measure the real DUT antenna performance anymore.

TGn channel model [8] is a correlation matrix-based model, but it contains also geometric description. It is possible to reconstruct TGn model to MIMO OTA environment with specified $\mathrm{Tx}$ (base station) antenna correlation matrices and specified, nongeometry-based, Doppler spectra. This 
can be done with the method of prefaded signals, but not with the method of plane wave synthesis. Both methods are discussed in the next section.

The clustered modelling principle of many MIMO channel models is a benefit for the method of prefaded signals. It is not a prerequisite, but it makes the method more efficient. With clustered channel models the individual rays are not essential, but the clusters they compose. In other words, it is not necessary to create a high number of rays with specific characteristics, but instead to model the sum effects, like spatial correlation, Doppler spectrum, power delay profile, XPR, and so forth, accurately.

\section{Synthesis of Propagation Environment}

This section describes two alternative methods to generate channel impulse response data (see Figure 2), to a fading emulator, to create desired radiated propagation environment within the test volume. The methods are prefaded signals synthesis and plane wave synthesis. Both methods can create statistically equal radiated propagation environment within the test volume. The plane wave synthesis has a point of view in individual rays (plane waves) while the prefaded signals synthesis focuses on clusters. The basic component created by the plane wave synthesis is a single plane wave with a specific angle of arrival, Doppler shift and magnitude. With the prefaded signals synthesis the basic component is a single cluster with parameters like nominal AoA, ASA, Doppler spectrum, and XPR. Methods are described in details in the following sub-sections.

4.1. Prefaded Signals Synthesis. Idea of the prefaded signals synthesis is to transmit Rayleigh or any other kind of faded signals separately from multiple OTA antennas. Fading sequences are created independently for each cluster. Clusters are mapped to OTA antennas based on cluster power angular spectrum (PAS) and OTA antenna directions. A single cluster is composed by a number of OTA antennas. Each OTA antenna contributing to the cluster has independent fading coefficient sequences with identical statistics. For example Doppler spectra of fading patterns of different OTA antennas are identical for a single cluster. Discrete PAS of the single cluster is formed by allocating power weights on top of OTA antennas i.i.d. fading patterns.

As a summary, Doppler spectrum, amplitude distribution, and Tx antenna and directional characteristics are created to the prefaded sequences. XPR and $\mathrm{Rx}$ directional characteristics are created by allocating i.i.d. prefaded sequences to a set of OTA antennas with specific power weights.

The principle of creating clusters, applying a number of OTA antennas, by superposition of independent fading patterns with direction-dependent powers approximate physical reality. We may consider OTA antennas as subareas of an scattering object creating the cluster. It is assumed that each subarea contributes to the cluster fading patterns with identical statistics. Assuming uncorrelated scattering (US) the subareas create independent fading patterns. Thus we can create the cluster effect with a set of spatially separated OTA antennas as far as the DUT antenna aperture is small with respect to the OTA antenna angular distance. More precisely, the angular resolution of DUT antenna array has to be smaller than $\Delta \theta$ in Figure 3.

This approach has at least one unrealistic element. In a real environment the Doppler spectrum should be angle dependent within the cluster. With the proposed method the Doppler spectrum is equal on all the OTA antennas composing the cluster. This effect may be observed by a DUT with directional antenna in the case of cluster with a high angular spread. We assume that this effect has negligible effect on DUT performance.

Details of creating fading patterns and power weights are described in the following. Here we present purely geometrybased method of creating Rayleigh fading coefficients. Anyhow it is possible to apply some other method too, as far as the $\mathrm{Rx}$ side directional and polarization characteristics are known. For example, noise filtering method can be applied for Rayleig fading. Also, for example, fluorescent light effects of TGn model can be included in the prefaded coefficients. The Doppler spectrum may be based on some other definition than geometric.

4.1.1. Per Antenna Fading Patterns. On the prefaded signals synthesis the fading coefficients are generated based on (5). Now the Rx antenna is unknown, thus $F_{r x}$ is substituted by an ideal OTA antenna pattern. The approximation is valid if we use OTA antennas with a high polarization isolation and a flat radiation pattern to the direction of the test zone. A Rayleigh fading pattern for cluster $n$ for a channel from Tx (BS) antenna $s$ to vertically polarized element of OTA antenna $k$ is

$$
\begin{aligned}
& H_{k, s, n}^{V}(t, \tau) \\
& =\gamma_{n} \sum_{m=1}^{M}\left[\begin{array}{l}
1 \\
0
\end{array}\right]^{T} \mathbf{A}\left[\begin{array}{l}
F_{t x, s, V}\left(\phi_{n, m}\right) \\
F_{t x, s, H}\left(\phi_{n, m}\right)
\end{array}\right] \\
& \cdot \exp \left(j 2 \pi v_{n, m} t\right) \delta\left(\tau-\tau_{n}\right) \sqrt{g_{k, n}},
\end{aligned}
$$

where $\gamma_{n}$ is an amplitude of cluster $n, \mathbf{A}$ is a polarization matrix and, $g_{k, n}$ is an antenna power weight. The fading pattern for the horizontally polarized element is obtained by changing $\mathrm{Rx}$ antenna pattern of $(9)$ to $\left[F_{r x, k, V} F_{r x, k, H}\right]=$ [0 1 1] as

$$
\begin{aligned}
& H_{k, s, n}^{H}(t, \tau) \\
& =\gamma_{n} \sum_{m=1}^{M}\left[\begin{array}{l}
0 \\
1
\end{array}\right]^{T} \mathbf{A}\left[\begin{array}{l}
F_{t x, s, V}\left(\phi_{n, m}\right) \\
F_{t x, s, H}\left(\phi_{n, m}\right)
\end{array}\right] \\
& \cdot \exp \left(j 2 \pi v_{n, m} t\right) \delta\left(\tau-\tau_{n}\right) \sqrt{g_{k, n}} .
\end{aligned}
$$

Antenna power weights determine, together with OTA antenna directions, a discrete power angular spectrum to the 
test area, as will be discussed subsequently. The polarization matrix

$$
\mathbf{A}=\left[\begin{array}{cc}
\frac{\exp \left(\Phi_{n, m, k}^{V V}\right)}{1+1 / \kappa_{n, m}^{V}} & \frac{\exp \left(\Phi_{n, m, k}^{V H}\right)}{1+\kappa_{n, m}^{H}} \\
\frac{\exp \left(\Phi_{n, m, k}^{H V}\right)}{1+\kappa_{n, m}^{V}} & \frac{\exp \left(\Phi_{n, m, k}^{H H}\right)}{1+1 / \kappa_{n, m}^{H}}
\end{array}\right]
$$

is composed of random, independent and identically distributed initial phases $\Phi \sim \operatorname{Uni}(0,2 \pi)$, and crosspolarization power ratios $\kappa^{V}$ and $\kappa^{H}$ for vertically and horizontally transmitted signals, respectively.

With a Rayleigh fading channel it is commonly assumed, for example in [10], that polarization components are independently fading [19] and polarization is practically always elliptical [20]. In the existing geometric channel models the XPR is specified, but the actual polarization states are not controlled. In MIMO OTA the propagation channel XPR is determined by coefficients $\kappa$ in (11). Strengths of radiated vertically and horizontally polarized fields are determined by channel coefficient $H_{k}^{V}(t)$ and $H_{k}^{H}(t)$.

The radio channel dimensions, like delay, Doppler, polarization, and Tx side spatial characteristics, are modelled by other terms of (9) than $g_{k, n}$. For DUT antennas, the essential dimension is Rx side PAS denoted as $P(\Omega)$, defined as power $P$ received from direction $\Omega$. OTA antennas power weights $g_{k, n}$ determine PAS around the test area. A method is discussed in the following to form a discrete PAS, with power weights, such that the resulting field follows the continuous PAS specified by the channel model.

4.1.2. Antenna Power Weights. Purpose of the MIMO OTA system is to create a specific propagation environment within the test area. A channel model specifies continuous PAS on RX side, which may be composed of a number of spatial clusters with PAS $P_{n}(\Omega)$. Typically $P_{n}(\Omega)$ is defined by a nominal AoA and a root mean squared (rms) angular spread. The shape of $P_{n}(\Omega)$ may follow, for example, uniform, Gaussian, or Laplacian functions. With a limited number of OTA antennas we can only approximate the continuous PAS.

A straight forward method to obtain power weights would be to define $g_{k, n}=P_{n}\left(\theta_{k}\right)$, where $\theta_{k}$ is direction of the $k$ th OTA antenna. This is not an optimal method, because it does not consider, for example, the size of the test area. This is analogous to a conventional filter design, where the method of sampling continuous impulse response does not produce optimal filter coefficients with a limited number of taps. A method to optimally utilize limited OTA antenna resources is needed.

The power angular spectrum is a Fourier transform pair with the spatial correlation function [21]. Instead of directly sampling the continuous PAS, it is beneficial to determine the discrete PAS by utilizing the spatial correlation function. Our proposed method to find optimal power weights is to sample the test area with an virtual array of ideal isotropic antennas. The target is to find power weights that minimize the mean squared error between theoretical correlations and correlations resulting from a discrete PAS, between elements of the virtual array.

Let us spatially sample the test area with a number of virtual antennas such that the antennas compose $M$ pairs. Theoretical correlation between $m$ th pair $(u, v)$ of antenna elements can be determined according to [20], omitting polarization, as

$$
\rho_{m}=\frac{\int F_{u}(\Omega) F_{v}^{*}(\Omega) P(\Omega) d \Omega}{\sqrt{\int\left|F_{u}(\Omega)\right|^{2} P(\Omega) d \Omega \cdot \int\left|F_{v}(\Omega)\right|^{2} P(\Omega) d \Omega}},
$$

where ()$^{*}$ denotes complex conjugate operation, $F_{u}$ and $F_{v}$ are complex radiation patterns of antennas $u$ and $v$, respectively, with a common phase center. For virtual antennas the amplitude $\left|F_{u}(\Omega)\right| \equiv 1$. Phase is determined by the wavelength and relative positions of elements $u$ and $v$.

Respectively, the correlation for $m$ th antenna pair $(u, v)$ with discrete PAS composed by $K$ OTA antennas in directions $\boldsymbol{\Theta}$ and with power weights $\mathbf{G}$ is

$$
\tilde{\rho}_{m}(\boldsymbol{\Theta}, \mathbf{G})=\frac{\sum_{k=1}^{K} F_{u}\left(\theta_{k}\right) F_{v}^{*}\left(\theta_{k}\right) g_{k}}{\sqrt{\sum_{k=1}^{K}\left|F_{u}\left(\theta_{k}\right)\right|^{2} g_{k} \cdot \sum_{k=1}^{K}\left|F_{v}\left(\theta_{k}\right)\right|^{2} g_{k}}},
$$

where $\boldsymbol{\Theta}=\left\{\theta_{k}\right\}, \theta_{k} \in[0,2 \pi]$ is a vector of OTA antenna directions and $\mathbf{G}=\left\{g_{k}\right\}, g_{k} \in[0,1]$, is a vector of OTA antenna power weights. The cost function to be minimized with respect to weights $\mathbf{G}$ for fixed directions $\boldsymbol{\Theta}$ is defined as

$$
E_{\rho}(\boldsymbol{\Theta}, \mathbf{G})=\sum_{m=1}^{M}\left|\rho_{m}-\tilde{\rho}_{m}(\boldsymbol{\Theta}, \mathbf{G})\right|^{2}
$$

Optimal vector of OTA antenna power weights G for (9) and (10), minimizing (14), can be solved applying some numerical optimization method. Polarization is omitted from the described method, because we assume identically shaped PAS for both vertical and horizontal polarizations. The assumption is aligned, for example, with $[10,13]$.

4.2. Plane Wave Synthesis. This section describes a method to generate channel impulse responses, to the system depicted in Figure 2, applying the plane wave synthesis. Components of the setup are shown in Figure 1. At first we describe the creation of a single static plane wave, from an arbitrary direction, with complex antenna weights. Then the method is extended for creation of frequency and spatially selective fading radio channel model.

The plane wave synthesis for MIMO OTA is an extension to disciplines of acoustics and electromagnetic plane wave synthesis [22]. The following principle is applied in the plane wave synthesis; a closed curve in 2D case or a closed surface in $3 \mathrm{D}$ determines the field within the curve/surface when no sources are present inside [22]. In other words, the right target field is generated inside the test zone, if such a field is generated, that the components of both the electric and magnetic fields tangential to the surface of the test zone field are equal to those of the target field [15].

Nyquist sampling of at least two samples per wavelength on edge of the test zone, implies an approximation of 


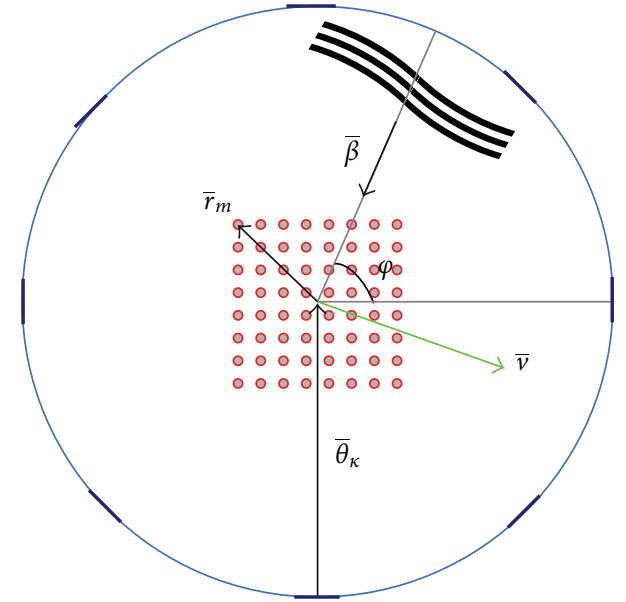

FIgure 4: A single plane wave from AoA $\varphi$ observed in location $\bar{r}_{m}$.

required probe numbers [15]. A waveform, observed within a test zone as a single plane wave from an arbitrary direction (angle of arrival AoA), can be created if the angular sampling by OTA antennas is dense enough. This is approximated by the rule of thumb of (1).

4.2.1. Single Static Plane Wave. As in Figure 4 a plane wave from an arbitrary AoA $\varphi$ is created by setting appropriate complex antenna weights $g_{k}$ to the CW (continuous wave on carrier frequency) transmitted from OTA antenna $k, k=$ $1, \ldots, K$. Each OTA antenna may contribute by radiating waves. Superposition of waves within the test area is then observed as a single plane wave with a specific AoA $\varphi$. Weights $g_{k}$ can be obtained, for example, by a numerical optimization. The numerical optimization may, for example, minimize the difference between the target field and the resulting field on the edge of the test area or some other sampling points within the test area. There are various procedures to perform the optimization. Here we present a method based on matrix inversion. The presented method is for vertically polarized ( $z$-polarized) fields only, but it can be extended to any polarizations.

Weights $g_{k}$ for a single plane wave can be obtained by solving OTA antenna weight vector $\mathbf{G}$ from the following matrix equation

$$
\mathrm{FG}=\mathrm{T},
$$

where:

(a) $\mathbf{F}=\left\{\alpha_{m, k}\right\} \in \mathbb{C}^{M \times K}$ is a transfer matrix of coefficients from $k$ th OTA antenna to $m$ th location $\bar{r}_{m}$;

(b) $\mathbf{G}=\left\{g_{k}\right\} \in \mathbb{C}^{K \times 1}$ is a vector of OTA antenna complex weights;

(c) $\mathbf{T}=\left\{e_{z}\left(\bar{r}_{m}\right)\right\} \in \mathbb{C}^{M \times 1}$ is a vector of complex target field values in locations $\bar{r}_{m}$ (of a plane wave with AoA $\varphi)$;

(d) $\bar{r}_{m}$ is a location vector, pointing from the origin to $m$ th sample point.
The transfer coefficient from $k$ th OTA antenna to $m$ th location $\bar{r}_{m}$ is composed of path loss term $L$ and a phase term as

$$
\alpha_{m, k}=L\left(d_{k, m}\right) \exp \left(-j\|\bar{\beta}\| d_{k, m}\right)
$$

where $d_{k, m}=\left\|\bar{\theta}_{k}+\bar{r}_{m}\right\|$ is the distance from the $k$ th OTA antenna to $m$ th location, $\bar{\theta}_{k}$ is a vector from $k$ th OTA antenna to centre of the test area, $\bar{\beta}$ is the wave vector pointing from AoA direction as in Figure $4,\|\bar{\beta}\|=2 \pi / \lambda_{0}$, and $\lambda_{0}$ is the wavelength at $f_{c}$. The target field for a plane wave is

$$
e_{z}\left(\bar{r}_{m}\right)=E_{0} \exp \left(-j \bar{\beta} \bullet \bar{r}_{m}\right)
$$

where field strength $E_{0} \equiv 1$ for the target field and $\bullet$ denotes the scalar product operation.

When both $\mathbf{F}$ and $\mathbf{T}$ are constructed the vector $\mathbf{G}$ can be solved. If $M=K$, the solution can be computed by matrix inversion

$$
\mathbf{G}=\mathbf{F}^{-1} \mathbf{T}
$$

In some cases $M>K$ sample points within the test area may be specified. This leads to an overdetermined optimization problem. In this case the target is to minimize squared L2 norm [23]

$$
\min _{\mathbf{G}}\|\mathbf{F G}-\mathbf{T}\|
$$

which results to solving $\mathbf{G}$ by the pseudo inverse

$$
\mathbf{G}=\left(\mathbf{F}^{H} \mathbf{F}\right)^{-1} \mathbf{F}^{H} \mathbf{T}
$$

Creation of vertical polarizations in 2D MIMO OTA configuration can be done with the discussed principles. For other polarizations or 3D environment the coefficient $\mathrm{G}$ have to be determined for three orthogonal polarizations instead of just one polarization. The mathematics to derive the cost function and the optimization for the multipolarized case is excluded from this paper.

4.2.2. Doppler Shift. In the previous we described how to find channel coefficients to create a single static plane wave with an arbitrary AoA. Now we introduce Doppler shift to the plane wave to enable time variant radio channels. A virtual motion of DUT creates Doppler shifts to plane waves. The Doppler shift to a plane wave with AoA $\varphi$ resulting from virtual motion to direction $\bar{\nu}$, as illustrated in Figure 4 , is

$$
\omega_{d}=-\bar{\beta} \bullet \bar{\nu}
$$

The Doppler shift can be introduced to the plane wave by multiplying Doppler frequency component $\omega_{d}\left(=2 \pi f_{d}\right)$ to the complex weights. That is by making the complex weights time dependent as follows:

$$
w_{k}(t)=g_{k} \exp \left(-j t \omega_{d}\right)
$$


Now the received field on an arbitrary location $m$ within the test area, neglecting the path loss, is

$$
\begin{aligned}
& E_{z}\left(t, \bar{r}_{m}\right) \\
& =\sum_{k=1}^{K} w_{k}(t) \exp \left(-j\left(\|\bar{\beta}\| d_{k, m}+t \omega_{c}\right)\right) \\
& =\sum_{k=1}^{K} g_{k} \exp \left(-j\|\bar{\beta}\| d_{k, m}\right) \exp \left(-j t\left(\omega_{d}+\omega_{c}\right)\right) .
\end{aligned}
$$

Even though the complex weights $w_{k}(t)$ are time dependent, the AoA dependent part $\left(g_{k}\right)$ has to be determined only once. Temporal behaviour is generated by multiplying fixed weight with rotating phasor as in (22).

4.2.3. Power Angular Spectrum. A specific power angular density function can be formed within the test zone by introducing a number of plane waves with an appropriate AoA and magnitude. The AoA range may be, for example, sampled uniformly, as in Figure 5(a), with $Q$ plane waves. Appropriate powers $P_{q}, q=1, \ldots, Q$, can be allocated to plane waves according to the target PAS. Another option is to fix powers and sample the angular space nonuniformly such that the target PAS is created as illustrated in Figure 5(b). In both cases each plane wave $q$ has a specific AoA $\varphi_{q}$ and power $P_{q}$. Also the Doppler shift $\omega_{d, q}$ will be unique for each plane wave depending on AoA and velocity vector of the virtual motion, as specified in (21).

Now the the complex weight of $k$ th OTA antenna will be a sum of contributions to $Q$ plane waves as follows:

$$
w_{k}(t)=\sum_{q=1}^{Q} \sqrt{P_{q}} g_{k, q} \exp \left(-j t \omega_{d, q}\right),
$$

where $g_{k, q}$ is solved separately for each plane wave $q$ by minimization of (19).

4.2.4. Delay Dispersion. Delay dispersion is straightforward to implement in the fading emulator-based setup. Assuming uncorrelated scattering (UC) the fading of each discrete delay component is independent. Thus channel coefficients for different delay taps are generated applying same principles as when creating a single plane wave and an arbitrary power angular spectrum.

Polarization characteristics, Tx (base station) antenna effects, and Tx side spatial characteristics can be included in the channel impulse responses generated with the plane wave synthesis. Description of the method to model the mentioned effects is excluded from this paper, but the principle is similar to the prefaded signals synthesis.

\section{Simulation Results}

Measurement and simulation results to verify the concept of anechoic chamber and fading emulator-based multiprobe MIMO OTA setup are reported in numerous COST 2100, COST IC1004 and 3GPP contributions. Paper [24] presents experimental and simulated results applying the prefaded signals method. Verification of a created radio channel with all its dimensions and characteristics is an extensive task. Here we show only a selection of simulation figures to demonstrate the two channel synthesis methods. The simulated characteristics are spatial correlation function (SCF), XPR, Doppler power spectrum (DPS), and temporal correlation function (TCF). Power delay profile (PDP) is excluded from the simulations, because creation of PDP is a trivial task with a digital fading emulator. Although the XPR simulation result illustrates a special case of a PDP.

In the simulations we had 16 uniformly spaced OTA antennas with $22.5^{\circ}$ angular spacing. OTA antennas were assumed as omnidirectional vertically polarized probes with an isotropic radiation pattern. Additional horizontally polarized probes, co-located with vertically polarized OTA probes, were present in the XPR simulation. Transmitter antenna (base station) was assumed as ideal omnidirectional vertically polarized element. In XPR case the target channel model contained nine equal power clusters, with $200 \mathrm{~ns}$ spacing in delay, and XPRs from $-20 \mathrm{~dB}$ to $+20 \mathrm{~dB}$ with a $5 \mathrm{~dB}$ increment. In the other cases the channel model was a single cluster model with AoA $=10^{\circ}$ and ASA $=15^{\circ}$. The direction of travel was $40^{\circ}$ and sampling was four samples per wavelength. At total 50,000 samples was simulated. An exception was the Doppler power spectrum and the temporal correlation, where 20 samples per wavelength and 250,000 samples was used to make the curves smoother.

5.1. Spatial Correlation. Figure 6 depicts the theoretical spatial correlation function for a Laplacian function shaped PAS and SCF calculated from channel impulse responses generated with the plane wave synthesis (PWS) and the prefaded signals synthesis (PFS). A time series of channel impulse responses for each OTA antenna was generated with the both methods. Then the field $E_{z}\left(t, \bar{r}_{m}\right)$ over time, along a segment of line of locations $\bar{r}_{m}$ was composed. Correlation between locations was calculated as the correlation between field coefficients $E_{z}\left(t, \bar{r}_{m}\right)$ in different locations over time. The examined correlation is an absolute value of a complex correlation coefficient. The simulation setup was similar to the measurement reported in [24].

Spatial correlation is an appropriate measure of the spatial dimension. Power angular spectrum observed by a DUT is inherently discontinuous and any power cannot be received from directions in between probes. Thus AoA or PAS estimation is not necessarily a meaningful technique to evaluate the created PAS. On the other hand, spatial correlation, as a fourier transform pair of PAS [21], is a continuous function and a good metric to assess the created spatial field.

The PWS curve (solid red) follows well the theoretical curve (blue circles) up to the simulated 1.5 wavelength spatial separation, as we can observe from the Figure 6. The PFS curve (solid green) follows the theoretical curve up to the simulated 1.2 wavelength spatial separation and deviates slightly after that. Anyhow the theoretical correlation on the area of deviation is low, 0.2 , or below, and the deviation 


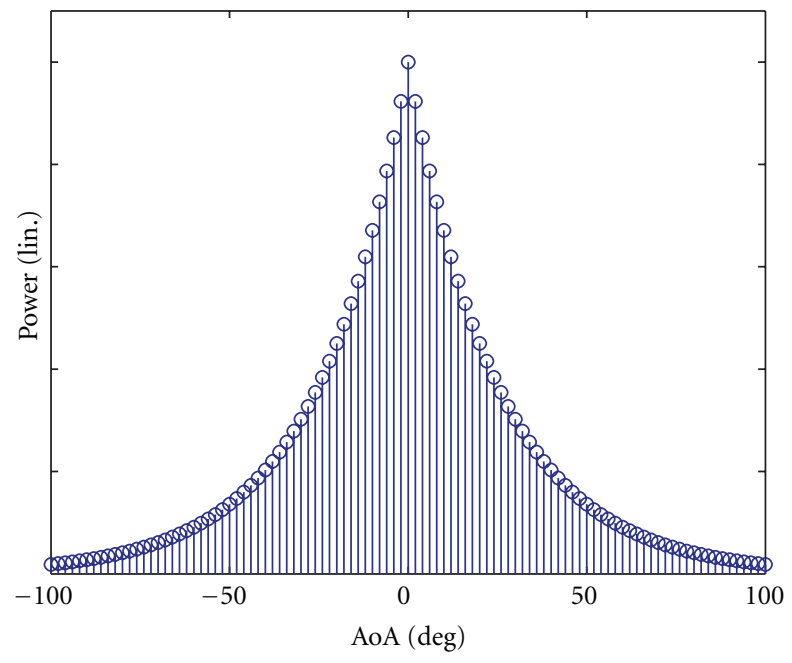

(a)

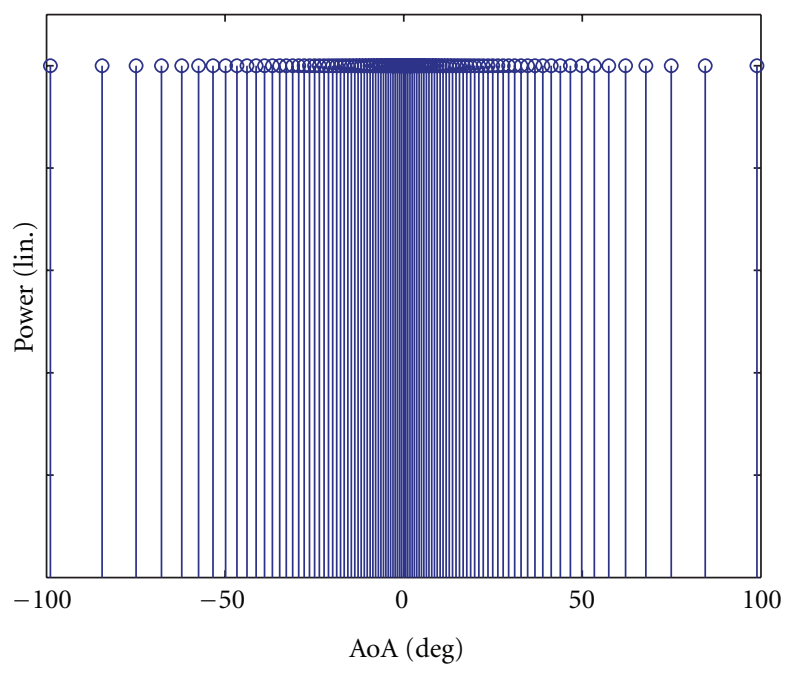

(b)

FIgURE 5: Uniform (a) and non-uniform (b) sampling of Laplacian function shaped PAS.

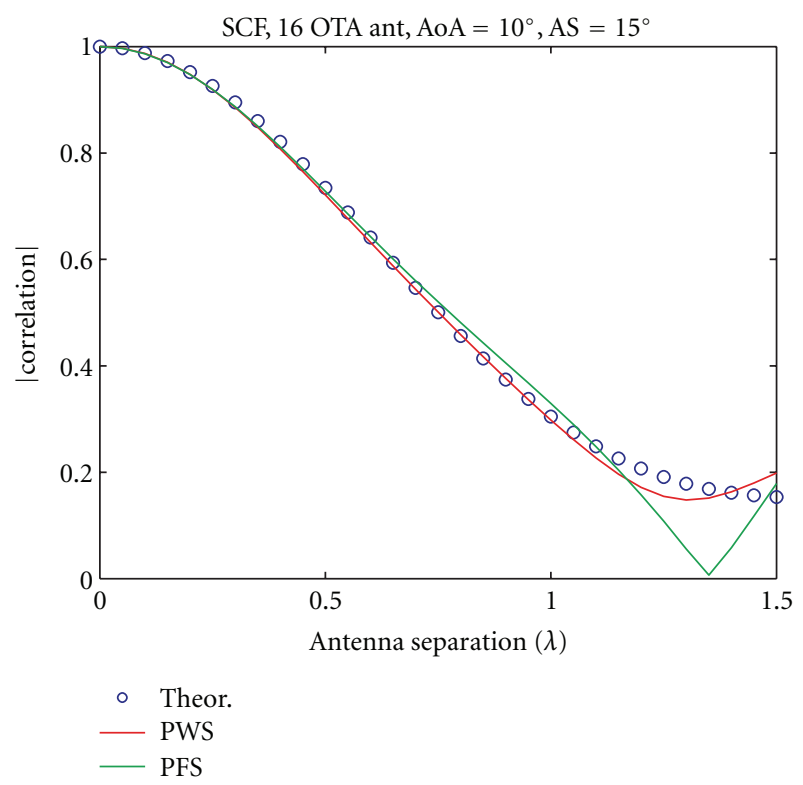

FIGURE 6: Simulated spatial correlations with 16 probes, $\mathrm{AoA}=10^{\circ}$, and ASA $=15^{\circ}$.

should have only marginal effect on, for example, channel capacity. We may conclude that the both channel mapping methods are capable of creating spatial radio channel characteristics according to the target model in the simulated case.

5.2. $X P R$. The polarization dimension is evaluated by investigating XPR values. With this type of geometric models the exact polarization state is not specified, as discussed earlier. Thus it is not feasible to simulate and compare polarization states. In the simulation a nine-cluster target model was defined, with different per cluster XPR values. The average power, received by an ideal vertically polarized DUT antenna, is the simulation result. Because Tx (base station) antenna was also vertically polarized ideal element, we get the simulated XPR directly by observing the $\mathrm{Rx}$ power and compensating out the path loss.

The simulated XPR values, achieved with the PFS method, and the target values are presented in the Figure 7. Also the deviation between target and simulation per cluster $\mathrm{XPR}$ values is shown. The maximum deviation in this simulation was below $0.2 \mathrm{~dB}$. In practical measurement the antenna nonidealities typically preclude this accurate results. The PWS method was excluded from the polarization simulation.

5.3. Doppler and Temporal Correlation. Simulated Doppler spectra from channel fading patterns generated with the PWS method (solid red) and the PFS method (solid green) are shown in Figure 8. The both spectra have similar spiky shape. Due to the nature of ray based geometric modelling it is difficult to compare the Doppler spectra directly. Especially any numerical comparison is practically impossible. Equivalent to the angular dimension, it is beneficial to transform spiky Doppler spectrum to a continuous domain of temporal correlation function.

TCF is a Fourier transform pair with the Doppler spectrum [21]. The transformation results to continuous function, which can be directly compared to a theoretical TCF. Such a comparison is depicted in the Figure 9, where real values of the complex temporal correlation coefficient are plotted. Temporal separation (delay) $\Delta \tau$ in the figure has a normalized unit of $1 / 2 f_{\max }$, where $f_{\max }$ is the maximum Doppler frequency component of the fading pattern. We can observe that the main lobe of the correlation resulting from both PWS (solid red) and PFS (solid green) methods follows almost perfectly the theoretical correlation (blue circles). PWS curve starts to deviate at the second peak of TCF while 


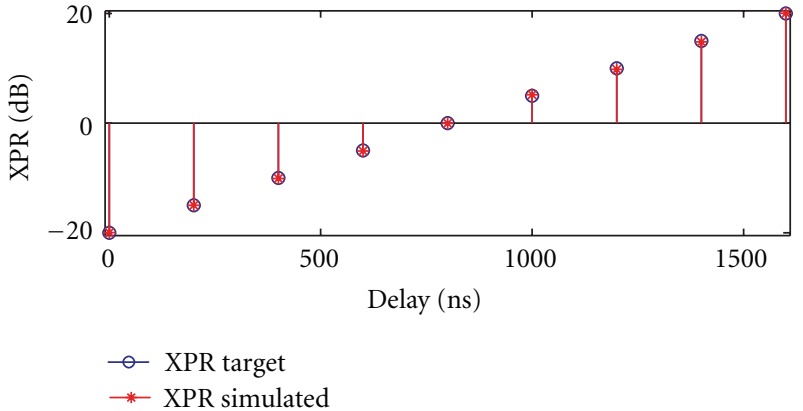

(a)

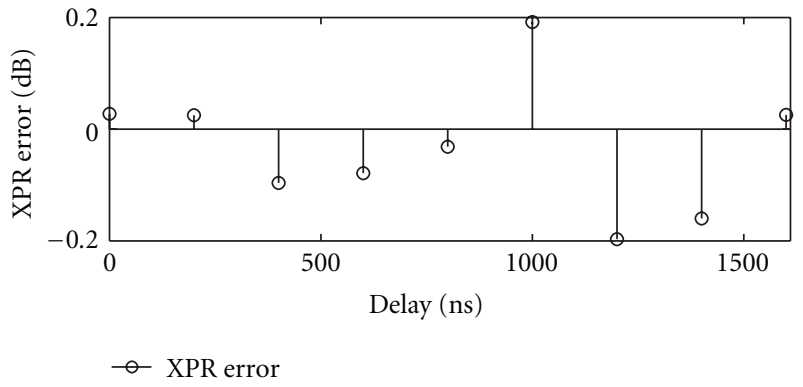

(b)

FIGURE 7: Simulated XPR with prefaded signals synthesis, target XPR is from -20 to $+20 \mathrm{~dB}$ with $5 \mathrm{~dB}$ increments.

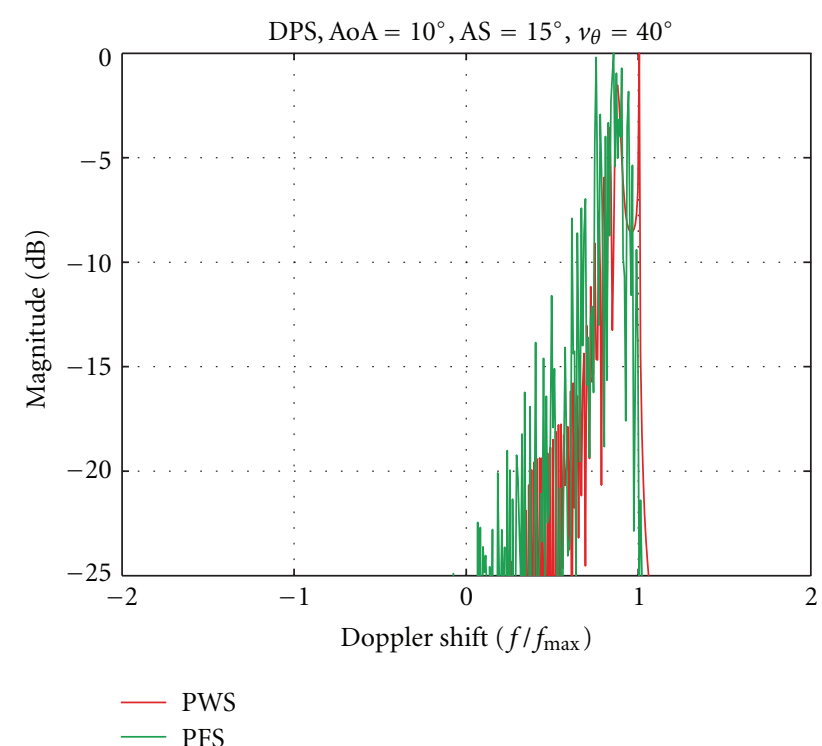

FIGURE 8: Simulated Doppler power spectra with 16 probes, AoA = $10^{\circ}$, and $\mathrm{ASA}=15^{\circ}$.

PFS curve has excellent match over the range of simulated $\Delta \tau$.

In the simulation we created clusters by a set of plain waves having uniform arrival angles. Arrival angles determine Doppler shifts by relation $v=f_{\max } \cos \left(\varphi-\theta_{v}\right)$. The selection of $1^{\circ}$ uniform angular sampling was probably not the optimal choice for creating accurate TCF. Some other angular sampling may have been better. Anyhow, the exact Doppler modelling is not the main focus of our contribution. The topic of defining parameters for a sum of rays based on temporal modelling is widely investigated by Pätzold, for example, in [25].

\section{Discussion}

In this paper we discussed the multiprobe MIMO OTA test system. We presented two alternatives to create radio propagation environment to MIMO OTA system, (1) prefaded approach and (2) plane wave approach. Both approaches

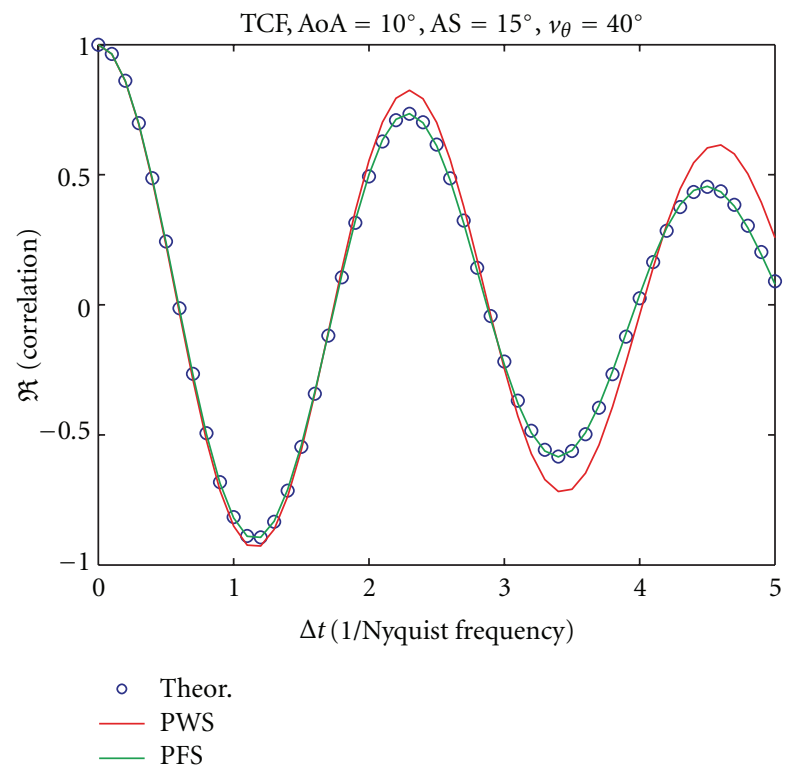

FIGURE 9: Simulated temporal correlations with 16 probes, AoA = $10^{\circ}$, and ASA $=15^{\circ}$.

are feasible to generate given radio propagation environment and we conclude the paper to highlight the pros and cons of each approach.

Benefits of the prefaded signals synthesis are; phase calibration is not required, simplicity, and possibility to create non-geometrical Doppler spectra. We recommend prefaded signals synthesis for standard models with wide clusters and Rayleigh fading or Ricean fading with a single LOS component. The LOS component, typically with a linear polarization state, can be created by rounding its AoA to the nearest dual polarized OTA probe. For that purpose the orthogonally polarized OTA antenna elements have to be colocated.

Main benefits of the plane wave synthesis for MIMO OTA compared to the prefaded signals synthesis are; support for LOS paths between OTA antennas, support for controlled polarization of paths (e.g., linear, circular, elliptic), and possibly more flexible support for dynamic (non stationary) 
channel models. Plane wave synthesis may also compensate distortion effect resulting from limited physical dimensions of an OTA antenna ring. These effects are, for example, distortion due to varying path loss and curved phase fronts, as discussed in [26]. A drawback of the plane wave synthesis is the requirement of phase calibration.

Complex weights $g_{k}$ for the plane wave synthesis are function of AoA and OTA antenna configuration only. For a fixed OTA antenna configuration it is possible to precalculate weights $g_{k}$, for example, for one degree grid of AoA values. In the actual generation of impulse response data the weights could be taken from a table and it would not be necessary to calculate them again. This would be a remarkable reduction of computing time in the case of dynamic channel models with time variant AoA and other propagation parameters.

Simulation results indicate that the spatial, polarimetric, and temporal dimensions of a target channel model can be created with the both methods. Delay dimension is a trivial modelling with a digital fading emulator applying either of PWS or PFS methods.

\section{Acknowledgments}

The research was partly conducted in the framework the CELTIC Project CP5-026 WINNER+ and partly of a research project, abbreviated MIMOTA, funded by the Finnish Funding Agency for Technology and Innovation (Tekes). The authors would like to acknowledge the contributions of their colleagues, especially Mr. Lassi Hentilä.

\section{References}

[1] M. D. Foegelle, "Over-the-air performance testing of wireless devices with multiple antennas," RF Design, pp. 44-52, 2006.

[2] http://www.cost2100.org/.

[3] R. Verdone and A. Zanella, Eds., Pervasive Mobile and Ambient Wireless Communications: COST Action 2100, Springer, 2012.

[4] http://ic1004.org/.

[5] Vodafone, "Proposed SID revision for study item: measurement of radiated performance for MIMO and multi-antenna reception for HSPA and LTE terminals," 3GPP TSG-RAN 54, RP-111754, Berlin, Germany, 2011.

[6] http://www.ctia.org/.

[7] P. Kyösti, J. Nuutinen, J. Kolu, and M. Falck, "Channel modelling for radiated testing of MIMO capable terminals," in Proceedings of the ICT-Mobile Summit, Santander, Spain, June 2009.

[8] V. Erceg, L. Schumacher, P. Kyritsi et al., "TGn channel models, IEEE P802.11 wireless LANs, http://www.802wirelessworld .com:8802/," Tech. Rep., IEEE, 2004.

[9] D. S. Baum, J. Hansen, G. del Galdo, M. Milojevic, J. Salo, and P. Kyösti, "An interim channel model for beyond-3G systems: extending the 3GPP spatial channel model (SCM)," in Proceedings of the IEEE 61st Vehicular Technology Conference (VTC '05), vol. 5, pp. 3132-3136, Stockholm, Sweden, May 2005.

[10] P. Kyösti, J. Meinilä, L. Hentilä et al., "IST-4-027756 WINNER II deliverable 1.1.2. v.1.2, WINNER II channel models," Tech. Rep. IST-WINNER2, 2007.
[11] "Guidelines for evaluation of radio interface technologies for IMTAdvanced, ITU-R Report M.2135,” Tech. Rep., 2008, ITU$\mathrm{R}$.

[12] J. P. Nuutinen, P. Kyösti, Y. Gao, and M. D. Foegelle, "On the MIMO OTA test system," in Proceedings of the 5th International ICST Conference on Communications and Networking in China (ChinaCom '10), Beijing, China, August 2010.

[13] 3GPP/3GPP2 TR 25.996 V6.1.0, "Spatial channel model for multiple input multiple output (MIMO) simulations," Tech. Rep., 2003, 3rd Generation Partnership Project.

[14] L. Hentilä, P. Kyösti, and J. Meinilä, "Elevation extension for a geometry-based radio channel model and its influence on MIMO antenna correlation and gain imbalance," in Proceedings of the 5th European Conference on Antennas and Propagation (EUCAP '11), pp. 2175-2179, Rome, Italy, April 2011.

[15] T. Laitinen, P. Kyösti, and J. Nuutinen, "On the number of OTA antenna elements for plane-wave synthesis," in Proceedings of the 4th European Conference on Antennas and Propagation (EuCAP '10), Barcelona, Spain, April 2010.

[16] T. Imai, Y. Okano, K. Koshiro, K. Saito, and S. Miura, "Theoretical analysis of adequate number of probe antennas in spatial channel emulator for mimo performance evaluation of mobile terminals," in Proceedings of the 4th European Conference on Antennas and Propagation (EuCAP '10), Barcelona, Spain, April 2010.

[17] M. Sonkki, V. Hovinen, D. Sánchez-Escuderos, E. Salonen, and M. Ferrando-Bataller, "Scattering properties of wideband dual-polarized vivaldi antenna for MIMO OTA," in COST IC1004 TD(12) 03058, Barcelona, Spain, 2012.

[18] P. Almers, E. Bonek, A. Burr et al., "Survey of channel and radio propagation models for wireless MIMO systems," Eurasip Journal on Wireless Communications and Networking, vol. 2007, Article ID 19070, 2007.

[19] S. R. Saunders, Antennas and Propagation for Wireless Communication Systems, John Wiley \& Sons, Chichester, UK, 1999.

[20] R. Vaughan and J. B. Andersen, Channels, Propagation and Antennas for Mobile Communications, IET, London, UK, 2003.

[21] M. Pätzold, "System function and characteristic quantities of spatial deterministic Gaussian uncorrelated scattering processes," in Proceedings of the 57th IEEE Semiannual Vehicular Technology Conference (VTC '03), pp. 256-261, April 2003.

[22] W. A. T. Kotterman, A. Heuberger, and R. S. Thomä, "On the accuracy of synthesised wave-fields in MIMO-OTA set-ups," in Proceedings of the 5th European Conference on Antennas and Propagation (EUCAP '11), pp. 2560-2564, Rome, Italy, April 2011.

[23] G. H. Golub and C. F. van Loan, Matrix Computations, The Johns Hopkins University Press, Baltimore, Md, USA, 3rd edition, 1996.

[24] P. Kyösti, J. P. Nuutinen, and T. Jämsä, "MIMO OTA test concept with experimental and simulated verification," in Proceedings of the 4th European Conference on Antennas and Propagation (EuCAP '10), Barcelona, Spain, April 2010.

[25] M. Pätzold, Mobile Fading Channels, John Wiley \& Sons, Chichester, UK, 2002.

[26] P. Kyösti and L. Hentilä, "Criteria for physical dimensions of MIMO OTA multi-probe test setup," in Proceedings of the 6th European Conference on Antennas and Propagation (EuCAP '12), Prague, Czech Republic, March 2012. 

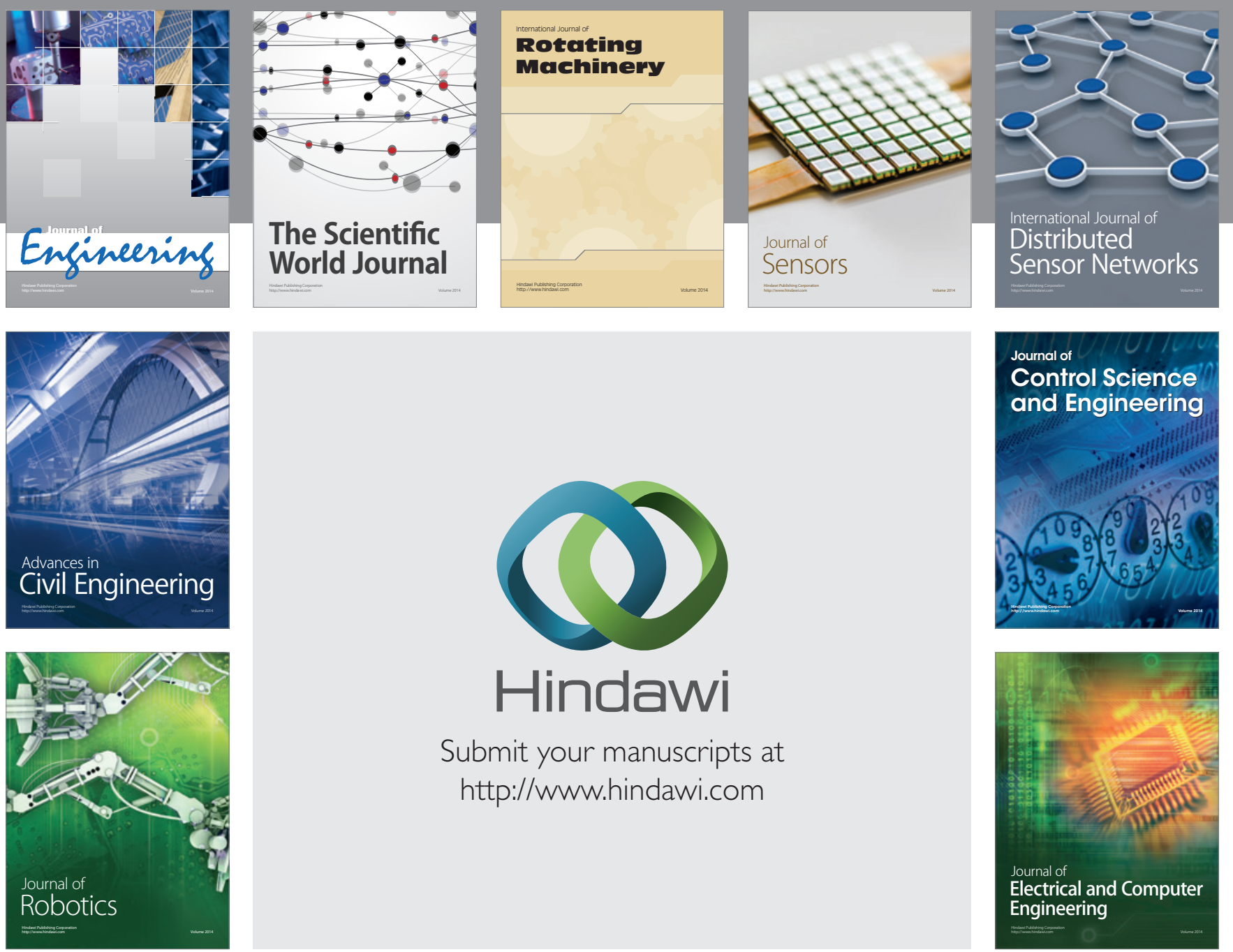

Submit your manuscripts at

http://www.hindawi.com
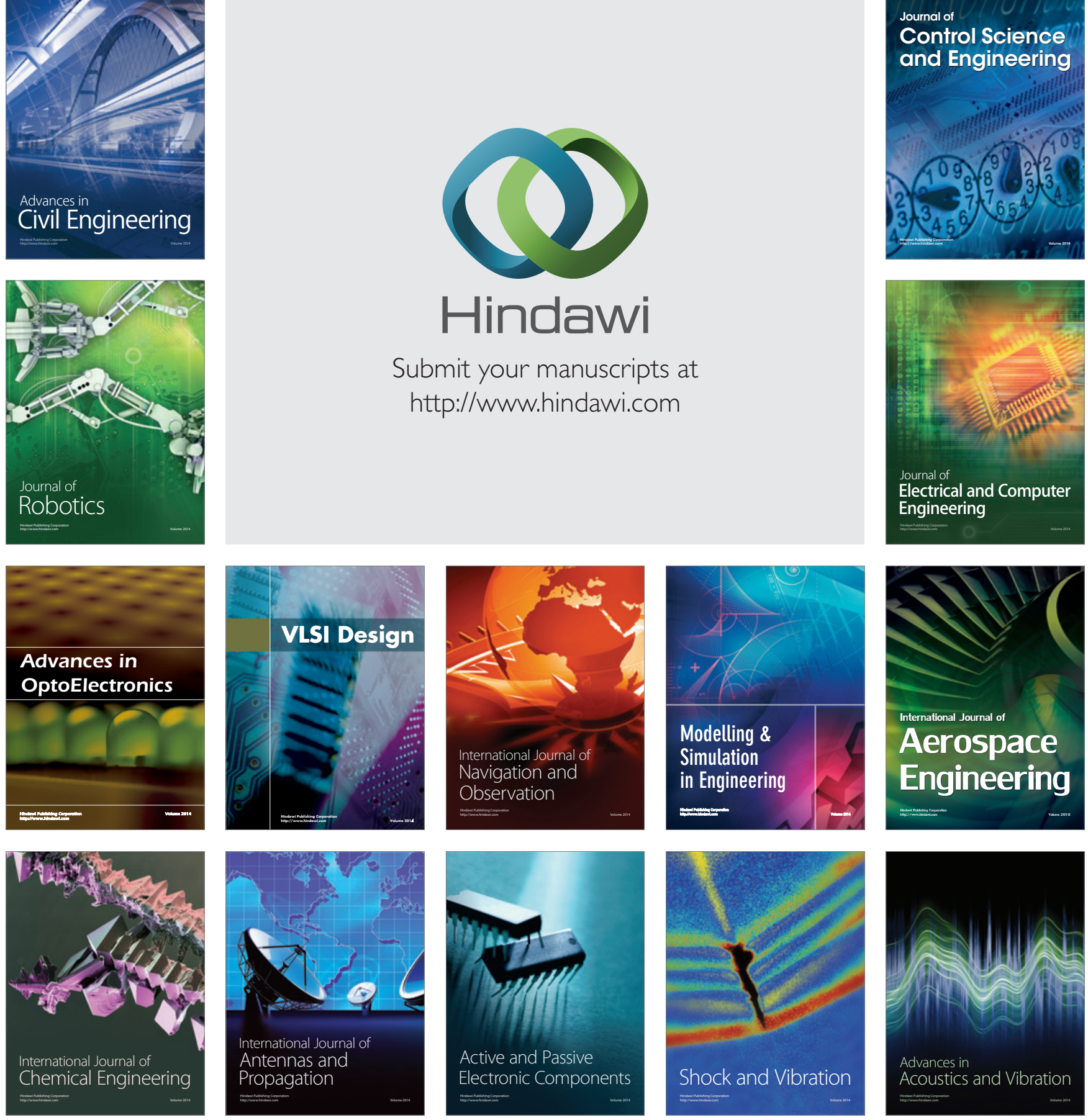